\title{
Primeros datos sobre la distribución de Myotis cf. nattereri y Myotis escalerai Cabrera, 1904 (Chiroptera: Vespertilionidae) en la Comunidad Autónoma de La Rioja.
}

\author{
Pablo Tomás AgirRe-Mendi ${ }^{1}$, CARlos IbÁÑEZ ${ }^{2}$
}

${ }^{1} \mathrm{c} /$ Paseo del río Grande, 7, $3^{\circ}$ - I; E - 01320 - Oyón-Oion, (Álava/Araba), Spain

${ }^{2}$ Estación Biológica de Doñana (CSIC) - c/ Americo Vespucio s/n E-41092, Sevilla, Spain

Correo electrónico del autor: leisleri@yahoo.es

DOI: http://dx.doi.org/10.14709/BarbJ.5.1.2012.02

English title: First data on the distribution of Myotis cf. nattereri and Myotis escalerai Cabrera, 1904 (Chiroptera: Vespertilionidae) in the Autonomous Community of La Rioja.

\begin{abstract}
This paper consists of a preliminary discussion of the chorology in the Autonomous Community of La Rioja (central northern Spain) of Myotis cf. nattereri and Myotis escalerai Cabrera, 1904, two recently segregated cryptic bat species. In all, 49 specimens from 11 localities in the northern sector of the mountains of the Sistema Ibérico and the Ebro river valley were identified by sequencing a fragment of approximately $700 \mathrm{bp}$ of the mitochondrial cytochrome b. The preliminary distribution of $M$. cf. nattereri in La Rioja is restricted to high- and mid-altitude areas of these mountains (altitudinal range: 850-1984 $\mathrm{m}$ a.s.1.), whereas M. escalerai is found in low- and mid-altitude areas of the region in an altitudinal range of 336-1150 $\mathrm{m}$ a.s.l. The morphological differences between the two species are presented.
\end{abstract}

Keywords: Myotis escalerai, Myotis cf. nattereri, Myotis sp. A, La Rioja, Distribution.

Resumen: Se presenta la distribución preliminar de Myotis cf. nattereri en 7 localidades de la Comunidad Autónoma de La Rioja, situadas en la zona central del sistema Ibérico Septentrional y de Myotis escalerai Cabrera, 1904 en 4 localidades distribuidas, tanto por el valle del río Ebro como en las sierras ibéricas. Se han identificado molecularmente 49 ejemplares de 11 localidades diferentes secuenciando un fragmento de unos $700 \mathrm{pb}$ del citocromo b. Aparentemente hay una importante segregación altitudinal entre ambas especies. El primer taxón presenta una distribución más restringida, ya que parece preferir las zonas de media y alta montaña, mejor conservadas y, en principio, por encima de los $850 \mathrm{~m}$ s.n.m. y hasta los $1984 \mathrm{~m}$ s.n.m. M. escalerai presenta, una distribución preliminar también restringida a las zonas de baja y media altitud de la región, evitando las áreas de mayor altitud, y su rango de altitud va desde 336 hasta los 1150 m s.n.m. Se ofrece información sobre diferencias morfológicas entre ambas especies.

Palabras clave: Myotis escalerai, Myotis cf. nattereri, Myotis sp. A, La Rioja, Distribución.

\section{INTRODUCCIÓN}

Recientemente utilizando diferentes marcadores moleculares se ha puesto en evidencia la ausencia en España de Myotis nattereri s.l. (Kuhl 1817), y en su lugar se ha confirmado la presencia de dos linajes genéticos, que representan a dos especies filogenéticas crípticas (Ibáñez et al. 2006; Salicini et al. 2011).

Myotis escalerai Cabrera, 1904, es la especie más abundante y frecuente en la Península Ibérica e Islas Baleares, con aparente preferencia bioclimática mediterránea amplia y un rango altitudinal que va desde el nivel del mar hasta los 1597 m s.n.m. en el Pirineo oscense (Ibáñez et al. 2006, Alcalde et al. 2008, Flaquer et al. 2010). Aunque también se ha encontrado en la vertiente norte de los Pirineos (Evin et al. 2009), se puede considerar como un endemismo ibérico. En coherencia con esta circunstancia el nombre común en castellano es murciélago ratonero ibérico (SECEMU, 2010).

La otra especie no ha sido descrita formalmente por lo que lo más correcto es llamarla provisionalmente Myotis cf. nattereri, aunque también aparece citado en numerosos artículos especializados como Myotis sp. A (Ibáñez et al. 2006, Mayer et al. 2007, García-Mudarra et al. 2009, 
Puechmaille et al., en prensa). Su área de distribución actual abarca la mitad norte de la Península Ibérica, la parte central y oriental de la vertiente norte de los Pirineos franceses y el tercio suroriental de Francia, la casi totalidad de Italia y el sur de Austria (Ibáñez et al. 2006, Mayer et al. 2007, Evin et al. 2009, Salicini et al. 2011, Puechmaille et al. en prensa). En España presenta un patrón de distribución restringido a las áreas de montaña de la mitad septentrional de la Península Ibérica, en general, a una altitud superior a 800 m s.n.m. excepto en la cornisa Cantábrica en donde probablemente llega al nivel del mar como indica su hallazgo en el Parque Natural as Fragas do Eume en A Coruña (Arzúa et al. 2010).

En La Rioja y localidades limítrofes se ha reportado la presencia de M. nattereri s.l. en diversas localidades (AgirreMendi \& Zaldívar 1991, Agirre-Mendi 1996 y 1998) antes de que se conociera la diversidad de este complejo específico. El principal objetivo de este trabajo es elucidar la situación específica del complejo "Myotis nattereri” en la Comunidad Autónoma de La Rioja, utilizando marcadores moleculares, y así contribuir al Atlas Mastozoológico de esta región.

\section{MATERIALES Y MÉTOdos}

\section{Area de estudio}

La Rioja es una pequeña región de algo más de $5000 \mathrm{~km}^{2}$ situada en el centro norte de Iberia en el valle del río Ebro. A pesar de su pequeño tamaño muestra una notable diversidad de paisajes y hábitats. Al norte de la región se encuentra el río Ebro con su altitud mínima de $260 \mathrm{~m}$ en el extremo noreste. Al sur de la región se encuentran las montañas del sistema Ibérico Septentrional con alturas que superan los 2200 $\mathrm{m}$ en el cuadrante suroeste. El clima y la vegetación están estrechamente relacionados con la altitud. Las zonas bajas tienen un clima típico mediterráneo con precipitación inferior a $600 \mathrm{~mm}$ y vegetación muy transformada por las actividades agrícolas. En las zonas de montaña las precipitaciones pueden superar los $1000 \mathrm{~mm}$ y existen extensos bosques de Quercus rotundifolia, Quercus faginea, Quercus pyrenaica, Fagus sylvaticus y Pinus sylvestris (Gobierno de La Rioja 2001).

\section{Muestreos}

Entre 1982 y 2011 se han realizado un total de 973 muestreos de murciélagos apropiados para detectar la presencia de ejemplares del complejo Myotis nattereri en 370 localidades diferentes que se ubican en su mayoría en La Rioja, pero también en algunas localidades limítrofes de Álava, Burgos y Soria. Los muestreos se han llevado a cabo con redes japonesas colocadas principalmente sobre superficies con agua y a la entrada de refugios nocturnos (cuevas, minas, etc.) o inspeccionando refugios diurnos.

Los resultados geográficos se ofrecen en cuadrículas U.T.M. de 10 por 10 kilómetros (Mapa 1), siguiendo el criterio de la Societas Europaea Mammalogica para este tipo de estudios.

\section{ANÁLISIS GENÉTICOS}

Con objeto de confirmar la identificación de los murciélagos de este complejo específico capturados en La Rioja, se ha secuenciado un fragmento de entre 700 y 768 bp de ADN mitocondrial (citocromo b) de 49 muestras provenientes de 11 localidades. En el caso de ejemplares de colecciones el ADN se ha extraído a partir de tejidos y en los individuos liberados a partir de dos biopsias de la membrana alar. El protocolo de extracción, amplificación y secuenciación es el de Ibáñez et al (2006). Algunas de las secuencias obtenidas están depositadas en el GenBank (Tabla1).

\section{RESULTADOS Y DisCUSión}

Como resultado de los muestreos específicos se han detectado la presencia de $M$. cf. nattereri/M.escalerai en 91 muestreos realizados entre 1987 y $2011 \quad(9,4 \%$ de los muestreos realizados), que corresponden a 63 localidades diferentes del área de estudio (51 en la Rioja, 9 en Burgos, 2 en Álava y 1 en Soria), distribuidas tanto por el valle del río Ebro como por las sierras ibéricas. En 11 de estas localidades se ha podido identificar molecularmente los ejemplares capturados (Tabla1). Solo en tres casos de estas 11 localidades se trata de agrupaciones más o menos estables (colonias de cría de Aradón y San Martín y posible "swarming” de San Cristóbal) que han sido visitadas y muestreadas en varias ocasiones. En los 8 casos restantes se trata de muestreos puntuales en los que se han capturado entre 1 y 10 ejemplares.

Los resultados genéticos indican la presencia de las dos especies en La Rioja (Tabla 1). Se confirma por primera vez por medio del análisis de marcadores moleculares la presencia de $M$. cf. nattereri y de $M$. escalerai en siete y cuatro localidades de La Rioja, respectivamente.

Con la información disponible, $M$. $c f$. nattereri se distribuye exclusivamente en la parte central de las sierras ibéricas riojanas, desde los 850 hasta los 1984 m s.n.m., aunque es previsible que ocupe áreas similares en la parte occidental y centro-oriental del sistema Ibérico Septentrional, y en las sierras de Toloño-Cantabria y Montes Obarenes (ver Mapa 1). M. cf. nattereri se ha capturado con redes japonesas en pleno bosque de hayas (2 localidades, La Agenzana y Las Bacarizas), en la entrada de cuevas (2) y simas (2) y en un collado de alta montaña con bosquetes de Pinus uncinata, en el Castillo de Vinuesa (Tabla 1). La mayoría de los datos corresponden a machos solitarios entrando a descansar en cavidades subterráneas con la excepción de la sima de San Cristóbal en donde puede haber un "swarming" de varias especies, aunque solo se han encontrado machos. Las dos únicas hembras capturadas en agosto en un hayedo con numerosas oquedades en los árboles estaban dando leche. Esta circunstancia unida a la ausencia de colonias de cría de M. cf. nattereri en los 684 muestreos realizados en edificios y cavidades subterráneas en La Rioja, sugiere fuertemente que $M$. cf nattereri evita cavidades subterráneas para criar y emplea para esta actividad huecos de árboles. En los muestreos realizados en cavidades $M$. cf nattereri se ha capturado junto con Rhinolophus hipposideros* (1), Myotis myotis* (5), M. emarginatus* (5), M. daubentonii* (3), M. mystacinus (1), M. alcathoe (1), M. bechsteinii* (2), Plecotus auritus* (5), P. austriacus* (2), Barbastella barbastellus (1) e Hypsugo savii (1); los señalados con asterisco corresponden a San Cristóbal. En los muestreos realizados en hayedo esta especie se ha capturado junto a $M$. mystacinus (2), $P$. auritus (4) y $B$. barbastellus (1), y en el collado de alta montaña con M. mystacinus (4), P. auritus (18), P. austriacus (1), B. barbastellus (1), H. savii (1) y Pipistrellus pipistrellus (1).

M. escalerai se distribuye en áreas de baja altitud de la parte centro-oriental del valle del Ebro y dos zonas de media altitud 
del sistema Ibérico Septentrional en las que abundan o no son raras las cavidades subterráneas (ver Mapa 1). Su rango de altitud va desde los 336 hasta los 1150 m s.n.m. y se ha capturado con redes solo en la entrada de refugios: dos cuevas, una mina y un túnel abandonado. Dos de estos refugios tenían colonias de cría, una en la mina de agua de Aradón (336 m s.n.m.) y otra en la cueva de San Martín (674 m s.n.m.), localizadas en el valle del Ebro y en el piedemonte de la sierra, respectivamente. En las otras localidades ubicadas en las sierras ibéricas, sólo se han capturado machos. El refugio de Aradón se ocupa en la época de cría y cuenta con una colonia de entre 70 y 150 adultos -machos y hembras- más crías. Este refugio está expuesto a molestias antropógenas por visitas incontroladas lo que se traduce que solo haya estado ocupado la mitad de los años que se ha visitado en el periodo 1992/2002. En este refugio también crían $R$. ferrumequinum, $R$. euryale, $M$. emarginatus y, ocasionalmente, Miniopterus schreibersii. La cueva de San Martín se ocupa solo en época de cría y la colonia se ha encontrado los 5 años en que se ha visitado entre 1991 y 2011. Cuenta con entre 100 y 400 individuos (adultos de ambos sexos más crías) y comparte refugio con una colonia de cría de $R$. euryale, así como individuos aislados de $R$. ferrumequinum, $R$. hipposideros, $P$. austriacus, B. barbastellus P. pipistrellus y M. schreibersii.

Aunque es probable que $M$. escalerai habite en otras áreas de la región de características bióticas similares no es una especie común ya que no ha sido encontrada ninguna colonia de cría en el resto de refugios subterráneos visitados que además han sido muestreados con redes en la boca de salida (ver mapa 1, anexo I).

Comparando en vivo ejemplares de las dos especies capturados simultáneamente en la Cueva de San Martín (M. escalerai) y Sima de San Cristóbal (M. cf nattereri), hemos encontrado una sustantiva diferencia morfológica entre ambos taxones en las cerdas que cubren el borde del uropatagio y que son características de todos los taxa del complejo "M. nattereri" (Dietz \& Helversen, 2004). En el caso de M. escalerai el aspecto general es que la capa formada por las cerdas es más densa. Esto es debido a la presencia en esta especie de una franja adicional de pelos en el borde del uropatagio que en vista ventral se dirige hacia el cuerpo del animal. Esta franja está ausente en $M$. cf. nattereri.

Esta diferencia se une a la mencionada por Cabrera (1904) en la descripción de $M$. escalerai relativa a la inserción del patagio en el tobillo que ha sido recuperada por Puechmaille et al. (en prensa). Además, según estos autores en $M$. escalerai, el borde del plagiopatagio en sus primeros 2-3 mm de longitud medidos desde el punto de inserción, se iría separando del tobillo de forma progresiva, es decir, formando en principio un ángulo muy pequeño, muy agudo con la línea que forman el pie y la tibia, que iría aumentando progresivamente. Sin embargo, en $M$. cf. nattereri el borde del plagiopatagio se separaría bruscamente desde su inserción en el dedo del pie, desde el primer milímetro, de la línea formada por el tobillo y la tibia, formando enseguida ambos un ángulo mucho más amplio que en el caso anterior, de unos $80^{\circ}$. Provisionalmente, proponemos que se utilice una combinación de los dos caracteres morfológicos mencionados para diferenciar a ambos taxones, en ausencia de análisis moleculares.
Por último, parece evidente que son necesarios más datos y por tanto más estudios de este tipo para determinar con precisión la distribución de ambas especies en La Rioja y para confirmar la validez de los caracteres morfológicos mencionados.

\section{Agradecimientos}

Juan Luis García-Mudarra e Irene Salicini han realizado los análisis genéticos.

\section{REFERENCIAS}

Agirre-Mendi, P.T., Zaldívar, C., 1991. Contribución al Atlas Mastozoológico de la Comunidad Autónoma de La Rioja I. Zubía. 9: 65-88.

Agirre-Mendi, P.T., 1996. Presencia de tres nuevos mamíferos silvestres (Mammalia, Vertebrata) en el territorio de la Comunidad Autónoma de La Rioja. Zubía. 14: 9-21.

Agirre-Mendi, P.T., 1998. Contribución al conocimiento de los murciélagos (Chiroptera, Mammalia) en la Comunidad Autónoma del País Vasco (Sierra de Cantabria). Zubía. 16: 61-90.

Alcalde, J.T., Trujillo, D., Artázcoz, A., Agirre-Mendi, P.T., 2008. Distribución y estado de conservación de los quirópteros en Aragón. Graellsia. 64(1): 3-16.

Arzúa, M., Hermida, R. J., Seage, R., Graña, D. A., Cerqueira, F., lamas, F. J., Conde, F., 2010. Resultados del inventario de quirópteros del Parque Natural as Fragas do Eume (A Coruña). III Jornadas de la SECEMU, métodos de seguimiento de murciélagos. A Coruña, 20 y 21 de noviembre de 2010.

CABrera, A., 1904. Ensayo monográfico sobre los quirópteros de España. Mem. Real Soc. Esp. Hist. Nat. 2 (5): 249287.

Dietz, C., von Helversen, O. 2004. Claves de Identificación Ilustradas de los Murciélagos de Europa. Vers. 1.0. Publicación Electrónica. Tuebingen \& Erlangen, Alemania. 1-72.

Evin, A., Lecoq, V, Durand, M.-O., Tillon, L., Pons, J.-M. 2009. A new species for the French bat list: Myotis escalerai (Chiroptera: Vespertilionidae). Mammalia. 73 (2): 142-144. DOI: https://dx.doi.org/10.1515/ MAMM.2009.030

Flaquer, C., Puig, X., Fàbregas, E., Guixe, D., Torre, I., RÀfols, R.G., PÁramo, F., Camprodón, J., Cumplido, J.M., Ruíz-JARILlo, R., LÓPEZ-BAuCElls, A., FreIXAS, L., Arrizabalaga, A. 2010. Revisión y aportación de datos sobre quirópteros de Cataluña: propuesta de lista roja. Galemys. 22 (1): 29-61.

García-MudarRa, J.L., IbÁÑEZ, C., Juste, J., 2009. The Straits of Gibraltar: barrier or bridge to Ibero-Moroccan bat diversity?. Biol. J. Linn. Soc. Lond. 96: 434-450. DOI: https://dx.doi.org/10.1111/j.1095-8312.2008.01128.x 
Gobierno De La Rioja, 2001. Medio Ambiente en La Rioja 2000. Consejería de Turismo y Medio Ambiente. Logroño, 1-194.

Ibáñez, C., García-Mudarra, J.L., Ruedi, M., Stadelmann, B., Juste, J., 2006. The Iberian contribution to cryptic diversity in European bats. Acta Chiropt. 8: 277-297. _DOI: http://dx.doi.org/10.3161/17335329(2006)8[277:TICTCD]2.0.CO;2

Mayer, F., Dietz, C., Kiefer, A., 2007. Molecular species identification boosts bat diversity. Front. Zool. 4: 4. DOI: https://dx.doi.org/10.1186/1742-9994-4-4

Puechmaille, S.J.,Allegrini, B., Boston,E.S.M.,DubourgSavage, M., Evin, A., Knochel, A., Le Bris, Y., LecoQ, V., Lemaire, M., Rist, D., Teeling, E.C., en prensa. Genetic analyses reveal further cryptic lineages within the Myotis nattereri species complex. Mammalian Biology - Zeitschrift für Säugetierkunde. DOI: https://dx.doi.org/doi:10.1016/j.mambio.2011.11.004
Salicini, I., IbáÑEz C., Juste J., 2011. Multilocus phylogeny and species delimitation within the Natterer's bat species complex in the Western Palearctic. Multilocus phylogeny and species delimitation within the Natterer's bat species complex in the Western Palearctic. Molecular Phylogenetics and Evolution. 61: 888-898.

SECEMU, 2010. Nombres de los murciélagos en España. Barbastella. 4: 15. DOI: https://dx.doi.org/10.1016/j. ympev.2011.08.010

\section{Anexo I Figuras y tablas}

\begin{tabular}{|c|c|c|c|c|c|c|c|c|c|}
\hline SP & TÉRMINO MUNICIPAL & LOCALIDAD & FECHA $(*)$ & UTM X & UTM Y & UTM Z & NoM & $\mathrm{N}^{\circ} \mathrm{H}$ & OBSERVACIONES (capturas, reproducción y GenBank) \\
\hline Mcfn & El Rasillo de Cameros & Cueva de Cerraúco & 07/08/1987 & 521558 & 4670138 & 1392 & 1 & & $2 \delta \delta$ \\
\hline Mcfn & El Rasillo de Cameros & Arroyo de la Agenzana & $13 / 08 / 1987$ & 523371 & 4672387 & 1273 & 1 & 1 & $1 \delta, 2$ 우, ambas dando leche \\
\hline Mcfn & El Rasillo de Cameros & Las Bacarizas & $24 / 08 / 1988$ & 521599 & 4670774 & 1345 & 1 & & $1 \delta$ adulto \\
\hline Mcfn & El Rasillo de Cameros & Sima de San Cristóbal & $17 / 08 / 2002$ & 519476 & 4670088 & 1519 & 17 & & ¿"swarming"? , 4 a 20 ơ ơ; DQ120885-6, JN591501 \\
\hline Mcfn & Nalda & Sima de la Hoya & $14 / 08 / 2003$ & 545292 & 4686313 & 1005 & 1 & & $1 \delta$ adulto \\
\hline Mcfn & Pradillo & Cueva de Peñamiel & $30 / 05 / 2008$ & 530427 & 4673033 & 850 & 1 & & $1 \delta$ adulto \\
\hline Mcfn & Villoslada de Cameros & Castillo de Vinuesa & $19 / 08 / 2009$ & 520794 & 4651838 & 1984 & 1 & & $1 \delta$ adulto \\
\hline Mesc & $S^{\text {ta. }}$ Engracia de Jubera & Cueva de San Martín & 18/09/1991 & $55 X X X X$ & $468 X X X X$ & 674 & 4 & 18 & Colonia de cría, 100 a 400 individuos; DQ120888, JN59148615 \\
\hline Mesc & Agoncillo & Mina de agua de Aradón & 06/06/1992 & $56 \times X X X$ & $469 \times X X X$ & 336 & & 1 & Colonia de cría, 70 a 150 individuos \\
\hline Mesc & Arnedillo & Túnel de Arnedillo & $25 / 08 / 1996$ & 565690 & 4673087 & 666 & 1 & & $4 \delta 3$ adultos; DQ120887 \\
\hline Mesc & Valgañón & Cueva de los Moros & 06/09/1996 & 493861 & 4685957 & 1150 & 1 & & $100^{x} 0^{\lambda}$ adultos \\
\hline
\end{tabular}

Tabla 1. Localidades de La Rioja en las que se ha encontrado a Myotis cf. nattereri (Mcfn) y Myotis escalerai (Mesc). $\underline{\boldsymbol{N}^{\boldsymbol{0}} \boldsymbol{M}}$, número de machos y, $\underline{\boldsymbol{N}^{\boldsymbol{o}} \boldsymbol{H}}$, número de hembras, identificados molecularmente. (*) Fecha del primer muestreo realizado en la localidad.

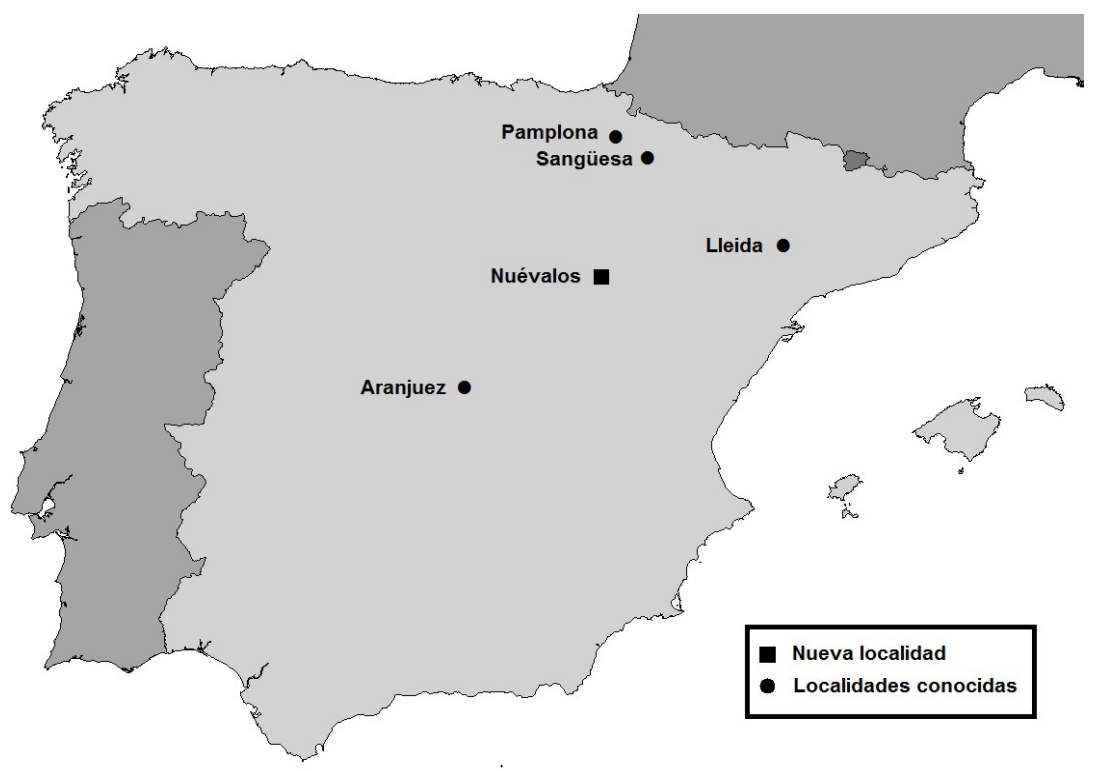

Fig. 1. Distribución de las localidades en las que se ha comprobado molecularmente la presencia de Myotis cf. nattereri (cuadrados verdes) y Myotis escalerai Cabrera, 1904 (triángulos rojos) en la Comunidad Autónoma de La Rioja. Curvas de nivel: en color amarillo, 400 m s.n.m., en pardo, 800 m s.n.m. y en verde, 1600 m s.n.m. 